\title{
Different quasispecies with great mutations hide in the same subgroup J field strain of avian leukosis virus
}

\author{
MAO YaQing ${ }^{1,2}$, LI WeiHua $^{3}$, DONG Xuan $^{3}$, LIU JinHua $^{1^{*}} \&$ ZHAO Peng ${ }^{3^{*}}$ \\ ${ }^{1}$ College of Veterinary Medicine, China Agricultural University, Beijing 100094, China; \\ ${ }^{2}$ China Institute of Veterinary Drug Control, Beijing 100081, China; \\ ${ }^{3}$ College of Veterinary Medicine, Shandong Agricultural University, Tai'an 271018, China
}

Received February 5, 2013; accepted March 25, 2013

\begin{abstract}
Blood samples were collected from a local strain of chickens associated with serious tumor cases in Shandong Province. The samples were inoculated into chicken embryo fibroblast and DF-1 cells for virus isolation and identification, respectively. The inoculated cells were screened for three common chicken tumor viruses. Nine strains of avian leukosis virus subgroup J (ALV-J) were identified, and were designated LY1201-LY1209. The env gene from the LY1201 strain was amplified and cloned. All nine resultant env clones (clones 01-09) were sequenced, and the gp85 and gp37 amino acid regions were subjected to homology analysis. Clones 01 and 03 had 10 amino acid deletions in the gp85 region compared to the other seven clones, suggesting that at least two quasispecies with obvious mutations coexist in the same field strain. Among these nine clones, three had identical gp85 and gp37 sequences, and were recognized as the dominant LY1201 quasispecies. The amino acid sequence homology of gp37 and gp85 among the nine clones was $98.5 \%-100.0 \%$ and $96.6 \%-100.0 \%$ respectively, suggesting that the gp85 region of the env gene can better display the quasispecies diversity of ALV-J than gp37.
\end{abstract}

avian leukosis virus subgroup J (ALV-J), gp85, gp37, quasispecies diversity

Citation: Mao Y Q, Li W H, Dong X, et al. Different quasispecies with great mutations hide in the same subgroup J field strain of avian leukosis virus. Sci China Life Sci, 2013, 56: 414-420, doi: 10.1007/s11427-013-4479-z

Avian leukosis virus (ALV) is a common avian retrovirus associated with neoplastic diseases. Based on host range, cross-neutralization, and viral interference, exogenous ALV in chickens is classified into A, B, C, D, and J subgroups, which induce different pathotypes of neoplastic diseases in chickens [1]. Among these subgroups, subgroups J, A, and $\mathrm{B}$ are the most prevalent in chickens from China [2-5].

Since it was first isolated and identified in the UK in 1988 [6], ALV-J quickly spread worldwide [7-11], causing severe economic losses in broiler farms in the 1990s and the early 2000s. In the mainland of China, ALV-J mainly caused myelocytomatosis (ML) and was officially recognized in white meat-type breeders in 1999 [4,5]. Clinical

*Corresponding author (email: zhaopeng@sdau.edu.cn; ljh@ cau.edu.cn) cases of ML tumors caused by ALV-J infections were reported in egg-type commercial layers in 2004 [12]. Because of successful global eradication programs, ALV-J-related tumor cases became very rare in white meat-type chicken farms. However, increasing numbers of tumor cases diagnosed as myelocytomatosis have been reported in local breeds of "yellow chickens" and other local breeds over the past few years in China [13-16].

In recent years, much attention has been paid to quasispecies diversity and the evolution of viruses $[17,18]$. Viral quasispecies have been correlated with gene duplications. For example, the chance of mutation for each nucleotide of the hemagglutinin (HA) gene of avian influenza virus (AIV), subtype H9N2, was calculated to be $2 \times 10^{-3}$ for each repli- 
cation, or about one base mutation occurring in every duplication cycle [19]. Therefore, RNA viruses in any cell culture or individual animal actually represent a viral population, composed of different quasispecies with some genomic differences. Because of the lack of correction mechanisms inherent in the RNA polymerase, ALV retroviruses are prone to mutation. Because ALV-J may contain a greater number of quasispecies than other subgroups, it is likely to be a good model for studying the quasispecies diversity of animal virus.

In the present study, nine highly pathogenic environmental ALV-J strains were isolated from serious tumor cases, and the quasispecies diversity was analyzed by nucleotide sequence comparisons of the envelope (env) gene from different clones from the same PCR product.

\section{Materials and methods}

\subsection{Sample animals}

In July of 2012, severe hemangioma tumors were detected in some chickens at a chicken farm in the city of Linyi, Shandong Province, China, with a total flock size of 8000 chickens. The chickens were all of a local breed named Huainanwang. The sick chickens had enlarged livers, spleens, and kidneys at 70 days of age, and the tumor-related mortality peaked between 90 and 110 days of age. The entire flock was eventually eliminated because of the infections.

\subsection{Cells and virus isolation}

DF-1 cells (a cell line from embryo fibroblast cells of C/E type chickens resistant to subgroup $\mathrm{E}$ endogenous ALV) were purchased from the American Type Culture Collection and used to isolate exogenous ALV. DMEM (pH 7.2; Invitrogen, Carlsbad, CA, USA) containing $100 \mathrm{U} \mathrm{mL}^{-1}$ penicillin and $100 \mu \mathrm{g} \mathrm{mL}^{-1}$ streptomycin was used as the basic medium. The growth medium consisted of the basic medium with the addition of $10 \%$ fetal bovine serum (FBS), while the maintenance medium contained $2 \%$ of FBS. Cell cultures were maintained in an incubator with $5 \% \mathrm{CO}_{2}$ at $37^{\circ} \mathrm{C}$.

Plasma samples were collected from nine randomly selected chickens and inoculated into chicken embryo fibroblast (CEF) and DF-1 monolayers, respectively, in 6-well culture plates. Inoculated DF-1 cultures were incubated for $12 \mathrm{~d}$. The supernatant from each well was then tested for ALV group-specific p27 antigen using an ALV p27 antigen detection kit (IDEXX laboratory, Beijing IDEXX Yuanheng Laboratories, Co., Ltd., Beijing, China) according to the manufacturer's instructions. The supernatants that were positive for p27 antigen were inoculated into a fresh DF-1 cell culture on a large scale to amplify virus stocks. Positive supernatant samples were stored at $-80^{\circ} \mathrm{C}$. Immunofluores- cence antibody assay (IFA) was used to test the cultured cells for ALV-J, ALV-A/B, REV, and MDV infection using ALV-J specific monoclonal antibody JE9, ALV-A/B specific chicken antiserum, reticuloendotheliosis (REV) specific monoclonal antibody 11B118, and Marek's disease virus (MDV) specific monoclonal antibodies BA4 and H19, respectively, as described by Qin et al. [20]. Primary antibody binding was detected using FITC-labeled anti-mouse IgG (Sigma-Aldrich, Saint Louis, MO, USA).

\subsection{Testing of antibody response to $\mathrm{REV}, \mathrm{ALV}-\mathrm{A} / \mathrm{B}$, and ALV-J}

Serum samples were collected from each of the same chickens used for virus isolations to examine the antibody response to ALV-A/B and ALV-J, using an ALV Antibody Test Kit for subgroups A/B and J (IDEXX). The serum was also assayed for antibodies to REV by ELISA using a Reticuloendotheliosis Virus Antibody Test Kit (IDEXX) according to the manufacturer's instructions. Each sample was tested twice to ensure the accuracy of the results.

\subsection{Amplification, cloning, and sequencing of the env gene region}

Genomic DNA was extracted from DF-1 cells infected with one ALV-J strain. To amplify a $2.2 \mathrm{~kb}$ fragment with the whole env gene of ALV-J, a pair of primers were used as env-F 5'-gctgccatcgagaggttact-3' and env-R 5'-agttgtcagggaatcgac- $3^{\prime}$, the PCR was performed as described by Wang and Cui [21]. Amplicons were agarose gel-purified using an E.Z.N.A Gel Extraction Kit (Omega Bio-Tek, Inc., Norcross, GA, USA), the gel-purified PCR products were cloned into pMD-18T plasmid vector (Takara Bio Inc., Tokyo, Japan), followed by propagation in DH5a (TIANGEN, Beijing, China). The transformants colonies were screened and identified by PCR identification with the primers introduced above. All positive clones were sequenced by a commercial sequencing service (Invitrogen, Shanghai, China). The nucleotide sequence alignment was carried out with the Clustal Method of the MegAlign program implemented in the DNAStar package ver. 7.01 (DNAStar Inc., Madison, WI, USA). The phylogenetic analysis was accomplished with Clustal W alignment and the neighbor-joining and maximum parsimony methods with 500 bootstrap replicates using the MEGA ver. 4.0 [22]. In total, nine clones were sequenced and analyzed.

\subsection{Homology analysis of gp85 amino acid sequences}

The amino acid sequences of the envelope protein, gp85, were used to determine the ALV subgroups. The gp85 sequences of the isolated viruses were compared with those of reference strains of ALV-J. Because the Shandong local chicken strains originated in Anhui Province, reference se- 
quences from 20 ALV-J strains isolated from Shandong and Anhui provinces, along with the ALV-J prototype HPRS103 sequence, were used as reference sequences for the homology analysis. The GenBank accession numbers of the sequences used are listed in Table 1 . The nucleotide sequence alignment was carried out by with the Clustal Method of the MegAlign program implemented in the DNAStar package ver. 7.01.

\section{Results}

\subsection{Isolation and identification of exogenous ALV}

$\mathrm{DF}-1$ cell cultures were incubated at $37^{\circ} \mathrm{C}$ for $12 \mathrm{~d}$, and the supernatants were tested for ALV p27 antigen. The results showed that all cultures inoculated with plasma samples from the nine chickens were positive for p27 antigen, indicating that they had exogenous ALV viremia. The virus isolates were designated LY1201-LY1209. IFA experiments showed that the infected cells could only be labeled with ALV-J specific monoclonal antibody, JE9, and showed no reaction with REV and MDV specific monoclonal antibodies (Figure 1). All serum samples were negative to antibodies against ALV-A/B and REV. More interestingly, the serum samples were also negative to ALV-J antibody, although they were all infected with ALV-J, as demonstrated in cell cultures. This may be caused by the immunosuppression induced by ALV-J early infection. As a result, the hosts failed to produce antibodies against ALV-J, which led to the persistent viremia in the chickens.

\subsection{Analysis of LY1201 quasispecies diversity based on gp85 and gp37 sequences}

Nine clones of the env gene from the LY1201 strain, designated clone 01-clone 09, were sequenced. The homology of the amino acid sequences of the gp85 region ranged from $96.6 \%-100.0 \%$ (Table 2), while homologies for the gp37 protein ranged from $98.5 \%-100.0 \%$ (Table 3). Ten amino acid deletions were found in the gp85 region (amino acids 210-219) of clones 01 and 03 compared to the other seven clones (Figure 2). The results indicated that at least two

Table 1 Reference ALV-J strains for comparison of gp85 amino acid sequences

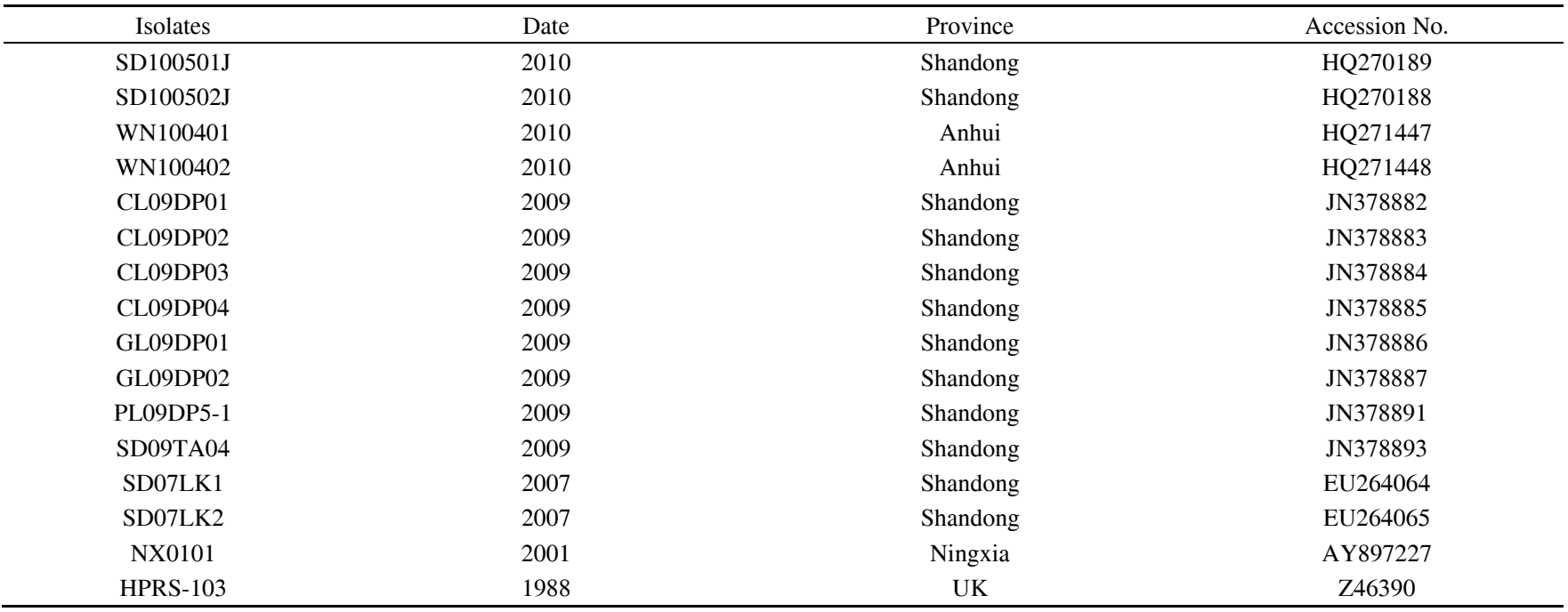

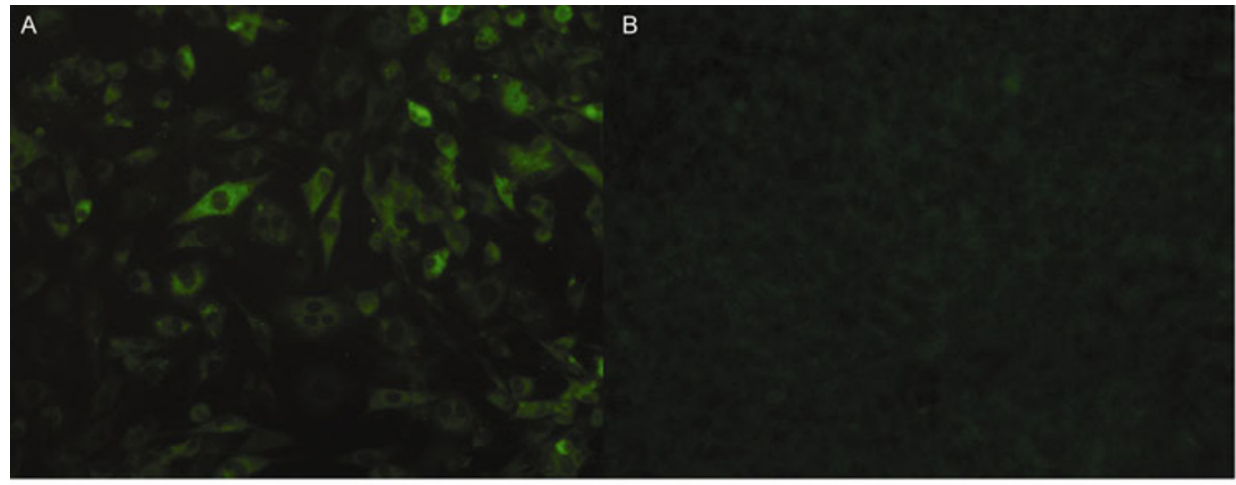

Figure 1 Detection of ALV-J in DF-1 cells by IFA with MAb JE9. A, Inoculated with LY1201. B, Uninfected DF-1 cells as blank control. 


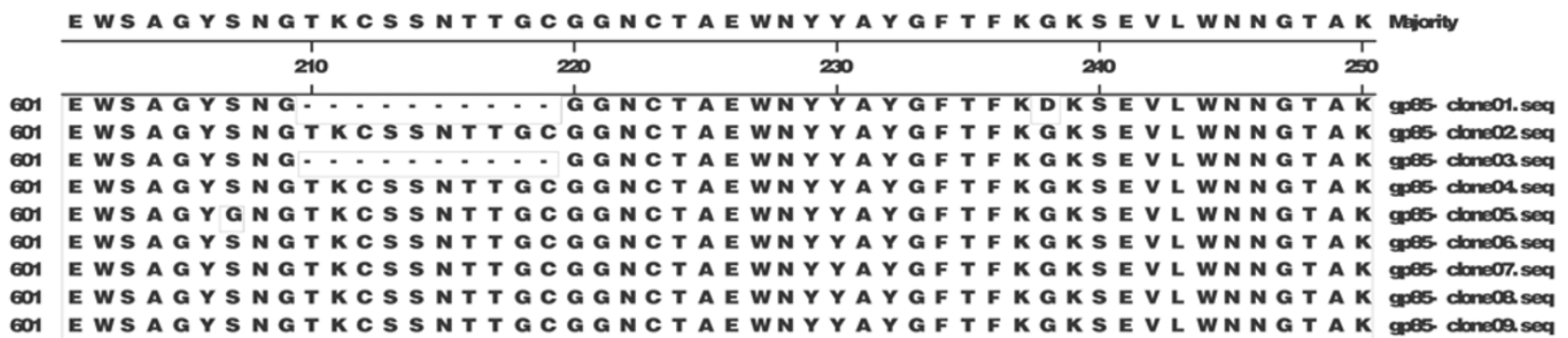

Figure 2 Comparison of deletions in the gp85 amino acid sequences of nine ALV-J env clones.

Table 2 Homology comparisons of the gp85 amino acid sequences of nine ALV-J env clones

\begin{tabular}{rccccccccc}
\hline & Clone01 & Clone02 & Clone03 & Clone04 & Clone05 & Clone06 & Clone07 & Clone08 & Clone09 \\
\hline Clone01 & 100.0 & 97.0 & 98.0 & 96.6 & 96.6 & 97.0 & 97.0 & 96.6 & 97.0 \\
Clone02 & & 100.0 & 98.3 & 99.0 & 99.7 & 100.0 & 100.0 & 99.7 & 100.0 \\
Clone03 & & & 100.0 & 98.0 & 98.0 & 98.3 & 98.3 & 98.0 & 98.3 \\
Clone04 & & & & 100.0 & 98.7 & 99.0 & 99.0 & 98.7 & 99.0 \\
Clone05 & & & & & 100.0 & 99.7 & 99.7 & 99.3 & 99.7 \\
Clone06 & & & & & & 100.0 & 100.0 & 99.7 & 100.0 \\
Clone07 & & & & & & & 100.0 & 99.7 & 100.0 \\
Clone08 & & & & & & & & 100.0 & 99.7 \\
Clone09 & & & & & & & & & \\
\hline
\end{tabular}

Table 3 Homology comparisons of the gp37 amino acid sequences of nine ALV-J env clones

\begin{tabular}{lccccccccc}
\hline & Clone01 & Clone02 & Clone03 & Clone04 & Clone05 & Clone06 & Clone07 & Clone08 & Clone09 \\
\hline Clone01 & 100.0 & 99.0 & 99.5 & 100.0 & 99.5 & 100.0 & 100.00 & 99.5 & 100.00 \\
Clone02 & & 100.0 & 98.5 & 99.0 & 98.5 & 99.0 & 99.0 & 98.5 & 99.0 \\
Clone03 & & & 100.0 & 99.5 & 99.0 & 99.5 & 99.5 & 99.0 & 99.5 \\
Clone04 & & & & 100.0 & 99.5 & 100.0 & 100.0 & 99.5 & 100.0 \\
Clone05 & & & & & 100.0 & 99.5 & 99.5 & 99.0 & 99.5 \\
Clone06 & & & & & & 100.0 & 100.0 & 99.5 & 100.0 \\
Clone07 & & & & & & & 100.0 & 99.5 & 100.0 \\
Clone08 & & & & & & & & 100.0 & 99.5 \\
Clone09 & & & & & & & & & 100.0 \\
\hline
\end{tabular}

quasispecies with obvious mutations coexist in the same ALV-J strain. Among these nine clones, three clones (clones 06, 07, and 09) had identical sequences in both the gp85 and gp37 regions, and were therefore recognized as the dominant LY1201 quasispecies. Taken together, these results suggested that the quasispecies diversity of ALV-J gp37 was much lower than that of gp85. The sequence differences of the gp85 region between different clones indicated that ALV-J strain LY1201 was composed of different quasispecies, despite the fact that these quasispecies shared a high level of homology.

\subsection{The gp85 protein comparison of LY1201 strain with ALV-J reference strains}

Clone 01, which had the 10 amino acid deletion, shared $85.5 \%-92.3 \%$ homology with the 20 reference strains in the gp85 amino acid sequence region. The phylogenetic tree based on the gp85 amino acid sequences showed that clone 01 was most closely related to reference strain PL09DP5-1 (data not shown). Another quasispecies, clone 06, which is the dominant quasispecies in this field strain, showed $85.7 \%-90.9 \%$ sequence homology to the reference strains in the gp85 amino acid region. The greatest numbers of amino acid mutations were identified in four areas of gp85: amino acids 110-120, 140-150, 190-200, and 210-220 (Figure 3).

\section{Discussion}

China has the second largest broiler chicken industry in the world, and all broiler breeds have been imported from different parts of the world over the last two decades. During the last 10 years, ALV-J has been reported globally [7-11]. ALV-J was officially recognized in China in 1999 [4,5], and 
DTDLAS QTACL I KALNTTLPWDPQELDI L GS QMI KNGT TRT C TF GS VCYKENNRSRVCHNF Miarity

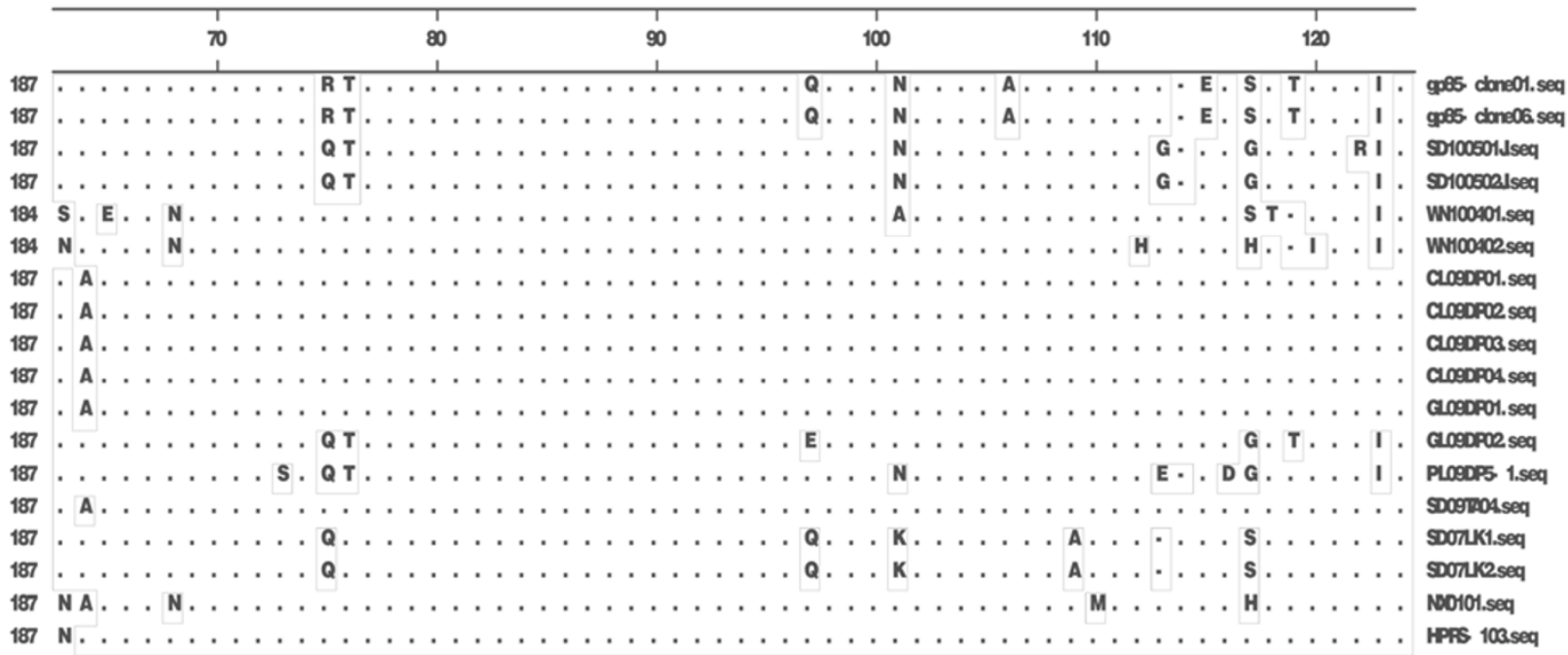

DGNF NGTGGAEAELRDF I AKWKS DDHLI RPY V QS WT MVSPI NTESFS I SSRYCGFTSNETR Majaity

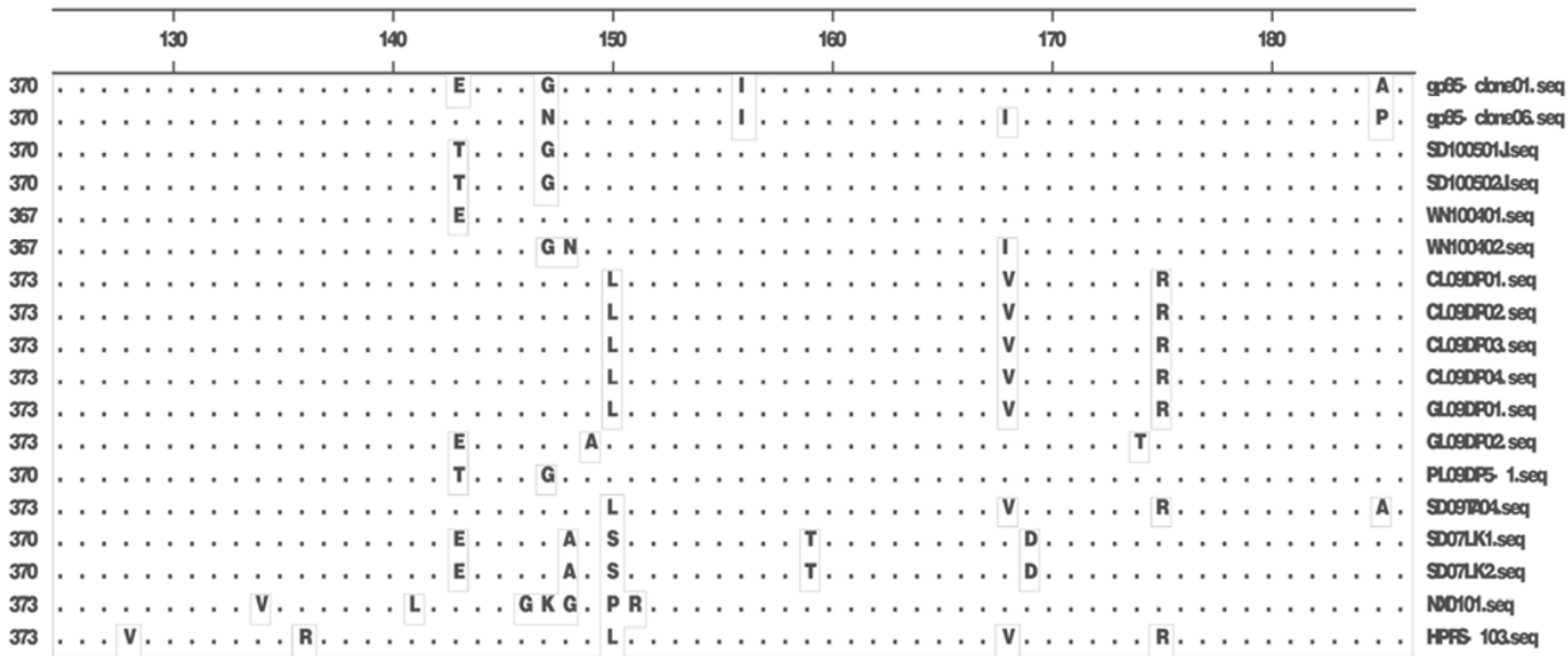

YYRGNLSNWCGSKGGE WSAGYSNGTKCSS NTTGCGGNCTAE WNYYAYGF TF GK QPE. . VL WN Mijity

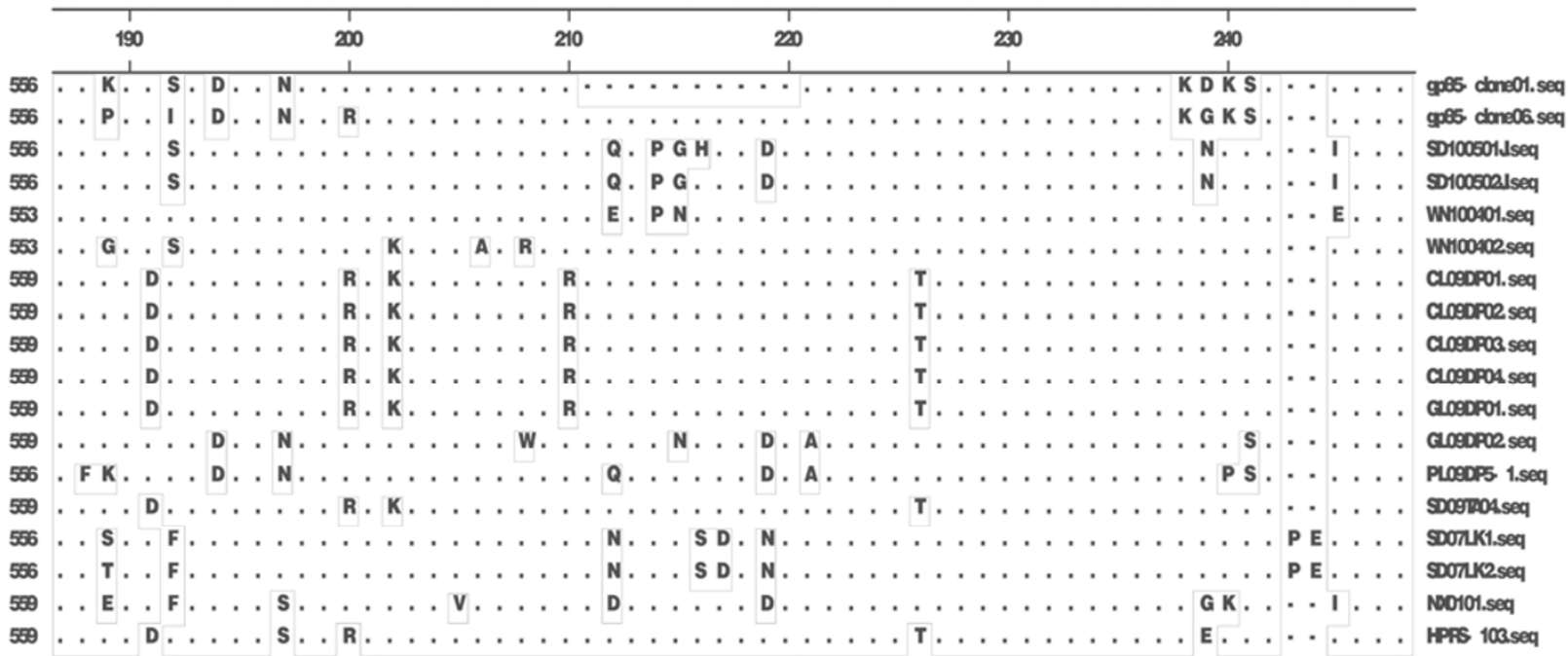

Figure 3 Amino acid alignment of quasispecies clones 01 and 06 with different reference gp 85 sequences. 
was reported in egg-type commercial layers in 2002 [12]. In addition, China has more than 40 local chicken breeds, which are also susceptible to infectious diseases, especially immunosuppressive viruses such as ALV and REV. Among these viruses, ALV-J is of particular concern for local chicken breeds, as our previous survey showed that the rates of ALV-J antibody in some local chicken strains were as high as $59.6 \%$ [23]. In the current study, nine highly pathogenic ALV-J strains were identified from a local chicken population suffering from tumors that were eventually fatal. This confirmed the serious risk of ALV-J to local breeds in China, and suggested that significant attention should be paid to the prevention and control of ALV-J in local chicken breeds.

The current study focused on the diversity and complexity of quasispecies of ALV-J isolates. As documented by previous reports, viral quasispecies usually be identified by analysis of quasispecies of some genes [18,24,25]. Therefore, to observe the quasispecies diversity of ALV-J, we can study the quasispecies diversity within some ALV-J genes, such as $e n v$, which has a high mutation rate and is associated with the antigen. The ALV env gene encodes two proteins, gp85 and gp37. However, gp85 is located on the surface of virions and is therefore the major protein for subgrouping of ALV.

Nine clones of the env gene from the LY1201 strain, designated clones 01-09, were sequenced and subjected to homology analysis. The gp85 amino acid region of the clones ranged from $96.6 \%-100.0 \%$, while that of the gp37 region, which is relatively conserved, was $98.5 \%-100.0 \%$. These differences between the clones revealed the presence of quasispecies, rather than being caused by mutations resulting from amplification or sequencing errors. A deletion of 10 amino acids was found in the gp85 region (amino acids 210-219) of clones 01 and 03, compared to the other seven clones (Figure 3), demonstrating that at least two quasispecies with obvious mutations coexist in the same ALV-J strain. These results indicated that the quasispecies diversity of ALV-J gp85 was much higher than that of gp37. The sequence differences among different clones in the gp85 region indicated that ALV-J field strain LY1201 was composed of multiple quasispecies, although these quasispecies shared a high level of homology.

Because RNA polymerases lack a proofreading mechanism, RNA viruses are prone to errors during replication. It is assumed that amino acid changes at certain sites of envelope glycoproteins could help mutated viruses evade the neutralizing activity of specific antibodies or immune cells. Mutants resistant to neutralizing antibodies may be able to replicate faster than parental viruses. These mutants would then gradually become the dominant quasispecies in the virus population. In this study, out of the nine clones, clones 06, 07, and 09 had identical amino acid sequences in both the gp85 and gp37 protein subunits, indicating that they belong to the same quasispecies. These quasispecies account for the majority of the LY1201 population. However, it is difficult to determine which quasispecies of this highly pathogenic field strain is more important for the pathogenesis: a quasispecies with 10 amino acid deletions, such as clone 01 , or the dominant quasispecies, represented by clone 06. Most likely the pathogenicity is due to the coexistence and cooperation of these two different quasispecies. The synergistic effects from coinfection of different avian immunosuppressive virus strains has been proved by many reports [26-28]. Whether the coinfection of different quasispecies can enhance pathogenicity requires further study.

It is important to note that the experimental design of the current study ensured that the observed differences among clones were a reflection of the presence of different quasispecies, rather than sequencing errors. In our previous studies, the ORF5 gene of porcine reproductive and respiratory syndrome virus (PRRSV) was amplified to show the differences between various quasispecies, while the conserved ORF6 gene was used as a control [18]. In the present study, only the env gene of the same field strain was amplified, and the PCR product was cloned to show the differences between the several ALV-J quasispecies. Because the env gene of ALV encodes two proteins (gp85, which mutates quickly, and the comparatively conserved gp37), if the differences between the clones were caused by sequencing errors, both gp85 and gp37 would give similar variation rates and patterns among different clones. The results also suggest that gp85 sequence regions can better display the quasispecies diversity of ALV-J than gp37.

The gp85 sequences of two major quasispecies, clones 01 and 06, were compared to different reference strains isolated from Shandong and Anhui provinces, China, from 2009 to 2010. Mutations were mainly located in four sequence regions: amino acids 110-120, 140-150, 190-200, and 210-220. Interestingly, in 2006 we established a model to study the evolution of ALV-J under antibody selective pressure. The results showed that amino acid regions 110-120, 140-150, and 188-193 were highly variable and more likely to be affected by antibody selective pressures. Therefore, we hypothesize that the mutations found in the current field strain are likely to be caused by antibody selective pressures, shown by the shared regions of highly variable amino acid sequence.

Although the clones investigated in this study came from the same PCR product of the same env gene, there was a fairly high level of variation in the gp85 sequences. However, the gp85 amino acid homology between the four reference strains, CL09DP01, CL09DP02, CL09DP03, and CL09DP04, was $100 \%$, and these reference strains were isolated from the same area. Therefore, this study is a good demonstration of the diversity and complexity of ALV-J quasispecies in different areas of China.

This work was financially supported by a special grant from Ministry of Agriculture of China in 2012. We thank Professor Zhao XiaoMin of Shandong Agricultural University for improving the English of the manuscript. 
1 Fadly A M, Nair V. Leukosis/sarcoma group. In: Diseases of Poultry. 12th ed. Ames: Blackwell Publishing, 2008. 514-568

2 Zhu M Z, Wu Y B, Cui Z Z. Isolation and identification of subgroup A avian leukosis virus from chickens of a local breed. Chin J Anim Infec Dis, 2009, 17: 31-35

3 Zhao D M, Zhang Q C, Cui Z Z. Isolation and identification of a subgroup $B$ avian leukosis virus from chickens of Chinese native breed Luhua. Chin J Virol, 2010, 26: 53-57

4 Du Y, Cui Z Z, Qin A J, et al. Isolation of subgroup J avian leukosis viruses and their partial sequence comparison. Chin J Virol, 2000, 16: 341-346

5 Cui Z, Du Y, Zhang Z, et al. Comparison of Chinese field strains of avian leukosis subgroup $J$ viruses with prototype strain HPRS-103 and United States strains. Avian Dis, 2003, 47: 1321-1330

6 Payne L N, Brown S R, Bumstead K, et al. A novel subgroup of exogenous avian leukosis virus in chickens. J Gen Virol, 1991, 72: 801-807

7 Fadly A M, Smith E J. Isolation and some characteristics of a subgroup J-like avian leukosis virus associated with myeloid leukosis ALV-J strain NX0101 decreases HI antibody titers to NDV in meat-type chickens in the United States. Avian Dis, 1999, 43: $391-400$

8 Thu W L, Wang C H. Phylogenetic analysis of subgroup J avian leucosis virus from broiler and native chickens in Taiwan during 2000-2002. J Vet Med Sci, 2003, 65: 325-328

9 Thapa B R, Omar A R, Arshad S S, et al. Detection of avian leukosis virus subgroup $\mathrm{J}$ in chicken flocks from Malaysia and their molecular characterization. Avian Pathol, 2004, 33: 359-363

10 Malkinson M, Banet-Noach C, Davidson I, et al. Comparison of serological and virological findings from subgroup $\mathrm{J}$ avian leukosis virus-infected neoplastic and non-neoplastic flocks in Israel. Avian Pathol, 2004, 33: 281-287

11 Lai H Z, Zhang H N, Ning Z Y, et al. Isolation and characterization of emerging subgroup $\mathrm{J}$ avian leukosis virus associated with hemangioma in egg-type chickens. Vet Microbiol, 2011, 151: 275-283

$12 \mathrm{Xu}$ B, Dong W, Yu C, et al. Occurrence of avian leukosis virus subgroup $\mathrm{J}$ in commercial layer flocks in China. Avian Pathol, 2004, 33: 13-17

13 Sun S H, Cui Z Z. Epidemiological and pathological studies of subgroup $\mathrm{J}$ avian leukosis virus infectious in Chinese local yellow chicken. Avian Pathol, 2007, 36: 221-226

14 Cheng Z Q, Zhang L, Liu S D, et al. Emerging of Avian leukosis virus subgroup $\mathrm{J}$ in a flock of Chinese local breed. Acta Micro Sin, 2005, 45: 584-587
15 Gao Y L, Shao H B, Luo Q P, et al. Molecular epidemiology of avian leukosis virus isolates from some regions of China in 2009. Chin J Prev Vet Med, 2010, 32: 32-36

16 Zhang H H, Liu Q, Qiu B, et al. Mixed infection of ALV-J and MDV in a flock of Shandong free range Chickens. Acta Vet et Zootech Sin, 2009, 40: 1215-1221

17 Richard S. Replicative homeostasis II: Influence of polymerase fidelity on RNA virus quasispecies biology: Implications for immune recognition, viral autoimmunity and other "virus receptor" diseases. Virol J, 2005, 2: 70

18 Zhao P, Ma C T, Dong X, et al. Evolution of quasispecies diversity for porcine reproductive and respiratory syndrome virus under antibody selective pressure. Sci China Life Sci, 2012, 55: 788-792

19 Liu H Q, Huang Y, Cheng J, et al. Genetic Mutations of the hemagglutinin Gene of H9N2 subtype avian influenza viruses under the selective pressure of vaccination. Chin J Virol, 2002, 18: 149-150

20 Qin A, Lee L F, Fadly A M, et al. Development and characterization of monoclonal antibodies to subgroup J avian leukosis. Avian Dis, 2001, 45: 938-945

21 Wang Z, Cui Z. Evolution of gp85 gene of subgroup J avian leukosis virus under the selective pressure of antibodies. Sci China Ser C-Life Sci, 2006, 49: 227-234

22 Tamura K, Dudley J, Nei M, et al. MEGA4: molecular evolutionary genetics analysis (mega) software version 4.0. Mol Biol Evol, 2007, 24: $1596-1599$

23 Zhao P, Cui Z Z, Ma C T, et al. Serological survey of the Avain leukosis virus infection in China native chicken flocks. J Anim Vet Adv, 2012, 11: 2584-2587

24 Töpfer A, Höper D, Blome S, et al. Sequencing approach to analyze the role of quasispecies for classical swine fever. Virology, 2013, 438: 14-19

25 Jardim A C, Bittar C, Matos R P, et al. Analysis of HCV quasispecies dynamic under selective pressure of combined therapy. BMC Infect Dis, 2013, 13: 61

26 Zhang Z, Zhuang G Q, Sun S H, et al. Pathogenesis of co-infection on broiler chicken by Marek's disease virus and reticuloendotheliosis virus. Acta Vet et Zootech Sin, 2005, 36: 62-65

27 Cui Z Z, Meng S S, Jiang S J, et al. Serological surveys of chicken anemia virus, avian reticuloendotheliosis virus and avian reovirus infections in white meat type Chickens in China. Acta Vet et Zootech Sin, 2006, 37: 152-157

28 Li Y P, Cui Z Z, Jiang S J, et al. Synergic inhibitory effect of co-infection of CAV and REV on immune responses to vaccines in SPF chickens. Chin J Vet Sci, 2008, 28: 1243-1246

Open Access This article is distributed under the terms of the Creative Commons Attribution License which permits any use, distribution, and reproduction in any medium, provided the original author(s) and source are credited. 